\title{
Reduced Order Modeling of delayed PEEC circuits
}

\author{
Francesco Ferranti*, Michel Nakhla ${ }^{\dagger}$, Giulio Antonini ${ }^{\ddagger}$, Tom Dhaene*, Luc Knockaert*, and Albert E. Ruehli ${ }^{\S}$ \\ ${ }^{*}$ Ghent University-IBBT, Department of Information Technology (INTEC), Ghent, 9000, Belgium \\ ${ }^{\dagger}$ Carleton University, Department of Electronics, Ottawa, Ontario, K1S 5B6, Canada \\ ‡Università degli Studi dell’Aquila, Dipartimento di Ingegneria Elettrica e dell’Informazione, L'Aquila, 67100, Italy \\ $\S$ Missouri University of Science and Technology, Electromagnetic Compatibility Laboratory, Rolla, Missouri, 65409, USA
}

\begin{abstract}
We propose a novel model order reduction technique that is able to accurately reduce electrically large systems with delay elements, which can be described by means of neutral delayed differential equations. It is based on an adaptive multipoint expansion and model order reduction of equivalent first order systems. The neutral delayed differential formulation is preserved in the reduced model. Pertinent numerical results validate the proposed model order reduction approach.

Index Terms-Delayed Partial Element Equivalent Circuit method, reduced order systems, neutral delayed differential equations.
\end{abstract}

\section{INTRODUCTION}

With the increase of operating frequencies, the accurate modeling of high-speed components and modern integrated circuits requires electromagnetic (EM) 3-D methods [1], [2] as necessary analysis and design tools. Large systems of equations are usually generated by the use of these methods and model order reduction (MOR) techniques are crucial to reduce the complexity of EM models and the computational cost of the simulations, while retaining the important physical features of the original system [3], [4]. Among all EM methods, the Partial Element Equivalent Circuit (PEEC) method [2] uses a circuit interpretation of the Electric Field Integral Equation (EFIE) [5], therefore it is especially suitable to problems involving both electromagnetic fields and circuits [2], [6]. If we consider a quasi-static PEEC formulation [2], which approximates the fullwave PEEC approach [7], an equivalent RLC circuit is obtained, since the time delays between the elements in the full-wave PEEC formulation are neglected. Systems of ordinary differential equations (ODE) are obtained. Standard MOR techniques for ODE systems can be used to reduce the size of a quasistatic PEEC model [3], [4]. When signal waveform rise times decrease and the corresponding frequency content increases or the geometric dimensions become electrically large, time delays must be taken into account and, therefore, included in the modeling.

A PEEC formulation which include delay elements, called $\tau$ PEEC [8], becomes necessary and leads to systems of neutral delayed differential equations (NDDE) [7]. While several successful MOR methods for large ODE systems have been proposed over the years, the reduction of large NDDE systems is still a very challenging research topic, since standard MOR techniques for ODE systems cannot be directly applied to NDDE systems. Especially, the reduction of electrically large structures where delays among coupled elements cannot be neglected or easily approximated by rational basis functions needs to be investigated and addressed.

Some techniques for the reduction of NDDE systems have been proposed [9]-[12]. In [9] an equivalent first order system is computed by means of a Taylor expansion, and then MOR Krylov subspace methods [3], [4] are applied. The NDDE formulation is not preserved in the reduction process. Multiple expansion points and the construction of rational local approximations of $\tau$ PEEC systems are used in [10]. Then, each rational local approximation is reduced by standard MOR methods and portions of these reduced rational models are combined to obtain a global reduced model for the frequency range of interest. As in [9], the NDDE formulation is not preserved in the reduction process. The approach in [11] extracts exponential terms (primary phase factors) and then the smoother remainders are expanded into a linear form and then projected to obtain the reduced model. Hence, the extraction of primary phase factors and the segregation of the system into multiple remainder phase matrices, each corresponding to a primary phase factor, are needed. In [12], an equivalent first order system is computed by means of a single point Taylor expansion [9] and a corresponding orthogonal projection matrix is computed by means of a block Arnoldi algorithm [3]. Then, an orthogonal projection matrix for the original NDDE system is extracted and a reduced NDDE system is obtained.

This paper presents a novel MOR method for $\tau$ PEEC models that is able to accurately reduce electrically large structures where delays among coupled elements cannot be neglected or easily approximated by rational basis functions. It is based on an adaptive multipoint expansion and MOR of equivalent first order systems [12]. The NDDE formulation is preserved in the reduced model.

The paper is organized as follows. Section II describes the modified nodal analysis (MNA) equations of the $\tau$ PEEC method. Section III describes the proposed MOR method for NDDE systems. Finally, a pertinent numerical example based on the $\tau$ PEEC method validates the proposed technique in Section IV.

\section{Delayed Peec formulation}

The PEEC method [2] stems from the integral equation form of Maxwell's equations. With respect to other EM methods, it is worth pointing out its capability to provide a circuit interpretation of the EFIE equation, thus allowing to handle 
complex problems involving both circuits and electromagnetic fields.

In the standard approach, volumes and surfaces are discretized into elementary regions, hexahedra and patches respectively [8] over which the current and charge densities are expanded into a series of basis functions.

Following the standard Galerkin's testing procedure, topological elements, namely nodes and branches are generated and electrical lumped elements are identified modeling both the magnetic and electric field coupling.

Conductors are modeled by their ohmic resistance, while dielectrics require modeling the polarization charge due to the dielectric polarization [13]. Magnetic and electric field coupling are modeled by partial inductances and coefficients of potential, respectively.

An example of $\tau$ PEEC circuit for a conductor elementary cell is illustrated, in the Laplace domain, in Fig. 1 where the current controlled voltage sources $s L_{p, i j} I_{j}$ and the charge controlled current sources $s Q_{i}$ model the magnetic and electric field couplings, respectively.

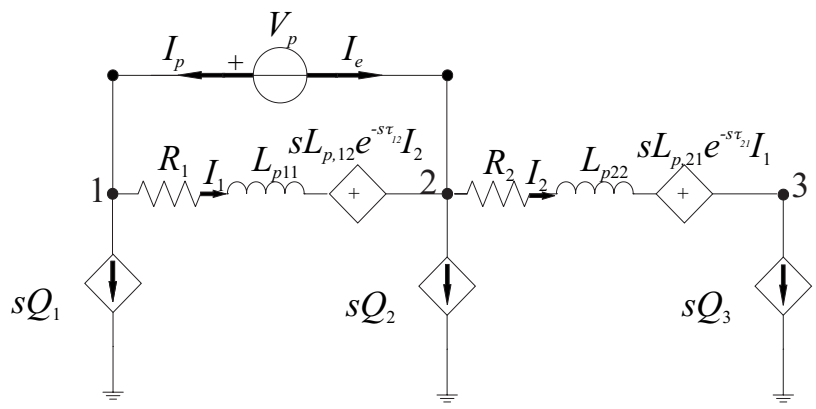

Fig. 1. Illustration of $\tau$ PEEC circuit electrical quantities for a conductor elementary cell.

Let us assume that the meshing process of conductors and dielectrics has generated $n_{i}$ volume cells where currents flow and $n_{n}$ surface cells where charge is located; the resultant number of elementary cells of conductors and dielectrics is $n_{c}$ and $n_{d}$, respectively and that of electrical nodes is $n_{n}$. Furthermore, let us assume to be interested in generating an admittance representation $\mathbf{Y}(s)$ having $n_{p}$ output currents $\mathbf{i}_{p}(t)$ under voltage excitation $\mathbf{v}_{p}(t)$. If the MNA approach [14] is used, the global number of unknowns is $n_{u}=n_{i}+n_{d}+n_{n}+n_{p}$ and an admittance representation of the PEEC circuit is obtained

$$
\begin{aligned}
\mathbf{C}(\boldsymbol{\tau}) \frac{d \mathbf{x}(t)}{d t} & =-\mathbf{G}(\boldsymbol{\tau}) \mathbf{x}(t)+\mathbf{B u}(t) \\
\mathbf{i}_{p}(t) & =\mathbf{L}^{T} \mathbf{x}(t)
\end{aligned}
$$

where $\mathbf{x}(t) \in \Re^{n_{u} \times 1}$ and $\boldsymbol{\tau} \in \Re^{n_{\tau} \times 1}$ contains all delays $\boldsymbol{\tau}_{L}, \boldsymbol{\tau}_{C}$, which denote the center-to-center delay matrices for the magnetic and electric field coupling. Since this is an $n_{p}$-port formulation, whereby the only sources are the voltage sources at the $n_{p}$-port nodes, $\mathbf{B}=\mathbf{L}$ where $\mathbf{B} \in \Re^{n_{u} \times n_{p}}$. Each delayed entry of matrices $\mathbf{C}(\boldsymbol{\tau})$ and $\mathbf{G}(\boldsymbol{\tau})$ act as a delay operator for the corresponding entry of vector $\mathbf{x}(t)$. Hence, (1) can be rewritten in the Laplace domain as:

$$
\begin{aligned}
s \mathbf{C}(s) \mathbf{X}(s) & =-\mathbf{G}(s) \mathbf{X}(s)+\mathbf{B} \mathbf{V}_{p}(s) \\
\mathbf{I}_{p}(s) & =\mathbf{B}^{T} \mathbf{X}(s) \\
\mathbf{C}(s) & =\mathbf{C}_{0}+\sum_{k=1}^{n_{\tau}} \mathbf{C}_{k} e^{-s \tau_{k}} \\
\mathbf{G}(s) & =\mathbf{G}_{0}+\sum_{k=1}^{n_{\tau}} \mathbf{G}_{k} e^{-s \tau_{k}}
\end{aligned}
$$

\section{DELAYED MOdel ORder REDUCTION}

In [12], an equivalent first order system is computed by means of a single point Taylor expansion [9] and a corresponding orthogonal projection matrix is computed by means of a block Arnoldi algorithm [3]. Then, an orthogonal projection matrix for the original NDDE system is extracted and a reduced NDDE system is obtained. The NDDE formulation is preserved in the reduction process. The proposed novel MOR algorithm is based on an adaptive multipoint expansion and MOR of equivalent first order systems. As in [12], the NDDE formulation is preserved. The multipoint feature allows to reduce electrically large structures with large delays $\left(2 \pi f r e q_{\max } \tau_{\max }>10\right)$ [11] that cannot be neglected or easily approximated by a single point expansion and rational functions. The equivalent first order system obtained after the single point Taylor expansion of exponential terms has an order equal to $q n_{u}$, where $q$ is the order of the Taylor expansion and $n_{u}$ the order of the original NDDE system [12]. Since exponential terms with large delays need many terms in the Taylor expansion to be accurately approximated, the reduction of equivalent first order systems becomes computationally expensive and sometimes not feasible. The multipoint expansion [15] addresses this issue and is able to accurately reduce NDDE systems with large delays, since a small expansion Taylor order can be used for each expansion point and the accuracy of the reduced model is increased by adding new expansion points. An adaptive algorithm is used to choose the expansion points, assuming that the order of the Taylor expansion is fixed for each expansion point. It is not described here due to the lack of space. At each expansion point, the MOR algorithm described in [12] is applied and the corresponding projection matrix $\mathbf{Q}_{i}, i=1, \ldots, n_{\text {points }}$ is computed. The final projection matrix $\mathbf{Q}$ is based on the orthogonalization of the stack column collection of all single expansion point projection matrices. The computation of $\mathbf{Q}$ in the case of multipoint expansion is explained in the flowchart in Fig. 2. The MOR algorithm described in [12] is called One point $D M O R$ in Fig. 2, where $n_{r}=q n_{p}$ represents the reduced order for each expansion point.

Once $\mathbf{Q}$ is computed, it is applied to the original NDDE system (2)-(3) and a reduced NDDE system 
Algorithm 1: $\operatorname{MultiPOINT}\left(N D D E_{\text {orig }},\left\{s_{k}\right\}_{k=1}^{n_{\text {points }}}, n_{r}, q\right)$

comment: Multipoint computation of basis $\mathbf{Q}$

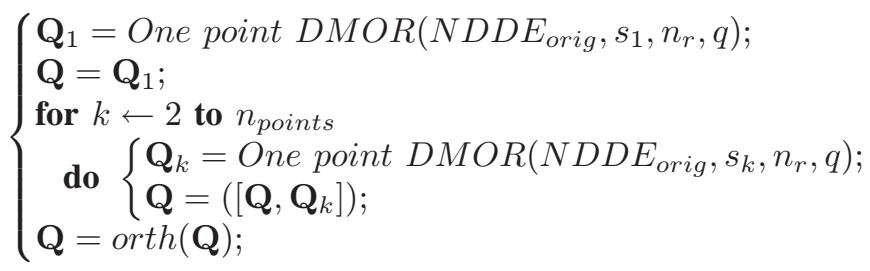

Fig. 2. Pseudocode for computing the orthogonal basis $\mathbf{Q}$ in the multipoint expansion case.

$$
\begin{aligned}
s \mathbf{C}_{r}(s) \boldsymbol{\chi}(s) & =-\mathbf{G}_{r}(s) \chi(s)+\mathbf{B}_{r} \mathbf{V}_{p}(s) \\
\mathbf{I}_{p}(s) & =\mathbf{B}_{r}^{T} \boldsymbol{\chi}(s) \\
\mathbf{C}_{r}(s) & =\mathbf{C}_{r, 0}+\sum_{k=1}^{n_{\tau}} \mathbf{C}_{r, k} e^{-s \tau_{k}} \\
\mathbf{G}_{r}(s) & =\mathbf{G}_{r, 0}+\sum_{k=1}^{n_{\tau}} \mathbf{G}_{r, k} e^{-s \tau_{k}}
\end{aligned}
$$

is obtained, where the following congruence transformations are used

$$
\begin{aligned}
\mathbf{C}_{r, i} & =\mathbf{Q}^{T} \mathbf{C}_{i} \mathbf{Q}, i=0, \ldots, n_{\tau} \\
\mathbf{G}_{r, i} & =\mathbf{Q}^{T} \mathbf{G}_{i} \mathbf{Q}, i=0, \ldots, n_{\tau} \\
\mathbf{B}_{r} & =\mathbf{Q}^{T} \mathbf{B} \\
\mathbf{L}_{r} & =\mathbf{Q}^{T} \mathbf{L}
\end{aligned}
$$

\section{NUMERICAL RESULTS}

Concerning the error criterion, let us define the weighted RMS-error as

$$
\begin{aligned}
& E r r= \\
& =\sqrt{\frac{\sum_{i=1}^{\left(n_{p}\right)^{2}} \sum_{k=1}^{K_{s}} \mid w_{Y_{i}}\left(s_{k}\right)\left(Y_{r, i}\left(s_{k}\right)-\left.Y_{i}\left(s_{k}\right)\right|^{2}\right.}{\left(n_{p}\right)^{2} K_{s}}}
\end{aligned}
$$

with

$$
w_{Y_{i}}(s)=\mid\left(Y_{i}(s)^{-1} \mid\right.
$$

It is used to assess the accuracy of reduced order models over the bandwidth of interest, which is sampled over $K_{s}$ samples.

\section{A. Multiconductor system}

A multiconductor system composed of four coplanar conductors with a length $\ell=10 \mathrm{~cm}$, a width $W=1 \mathrm{~mm}$, a thickness $t=1 \mathrm{~mm}$ and an horizontal spacing $S_{x}=3 \mathrm{~mm}$ has been modeled in this example. Fig. 3 shows its cross section. The system is analyzed on the frequency range $[0.0001,10] \mathrm{GHz}$.

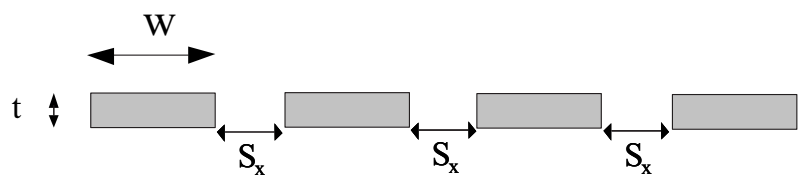

Fig. 3. Cross section of the multiconductor system.

The six ports of the system are defined between successive conductors.

The order of the original $\tau$ PEEC model is equal to $n_{u}=$ 2390 , the number of delays is equal to 632 and the largest delay gives $2 \pi f r e q_{\max } \tau_{\max }=21$. Table I shows some parameters of the proposed MOR method.

TABLE I

MOR PARAMETERS.

\begin{tabular}{|l|c|}
\hline Parameter & Adaptive method \\
\hline Taylor terms & 2 \\
Expansion points & 7 \\
Reduced order & 84 \\
Weighted RMS-error reduced model & 0.025 \\
\hline
\end{tabular}

Figs. 4-7 compare the magnitude and phase of the original and reduced NDDE model of $\mathbf{Y}_{33}(s)$ and $\mathbf{Y}_{36}(s)$. As clearly seen, the presented MOR method can reduce a large NDDE system with large delay terms, while accurately preserving the behavior of the original system.

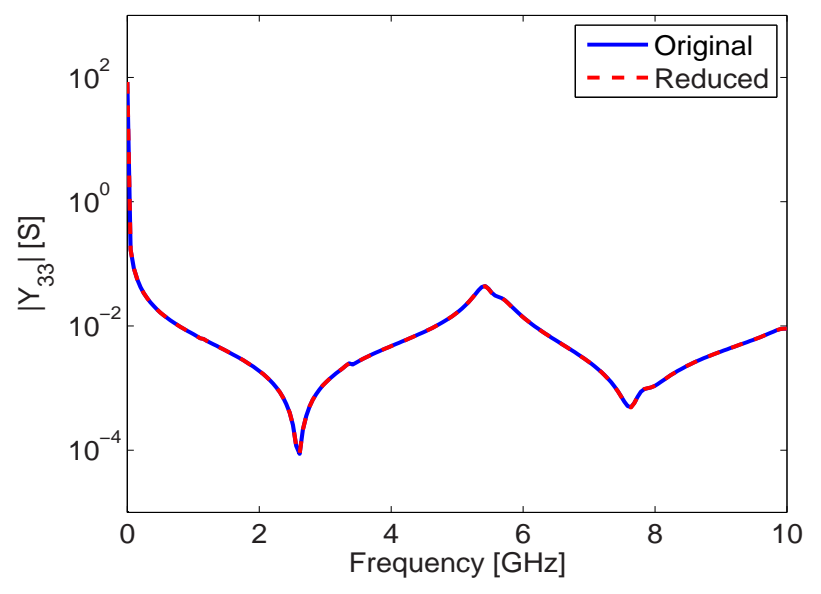

Fig. 4. Magnitude of $\mathbf{Y}_{33}$.

\section{CONCLUSiOnS}

We have presented a new MOR technique for large NDDE systems, which is applicable to $\tau$ PEEC models. It is able to accurately reduce electrically large structures where delays among coupled elements cannot be neglected or easily approximated by rational basis functions. It is based on an adaptive multipoint expansion and MOR of equivalent first order systems. The NDDE formulation is preserved in the 


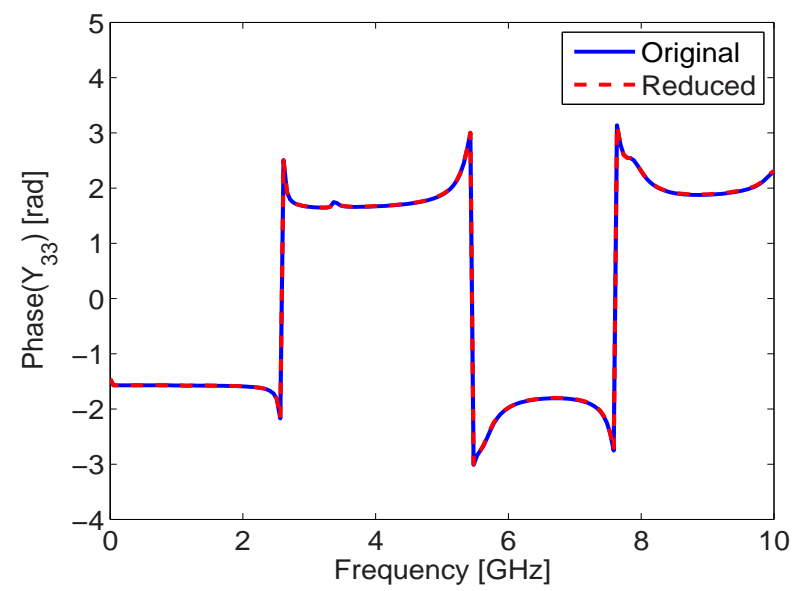

Fig. 5. Phase of $\mathbf{Y}_{33}$.

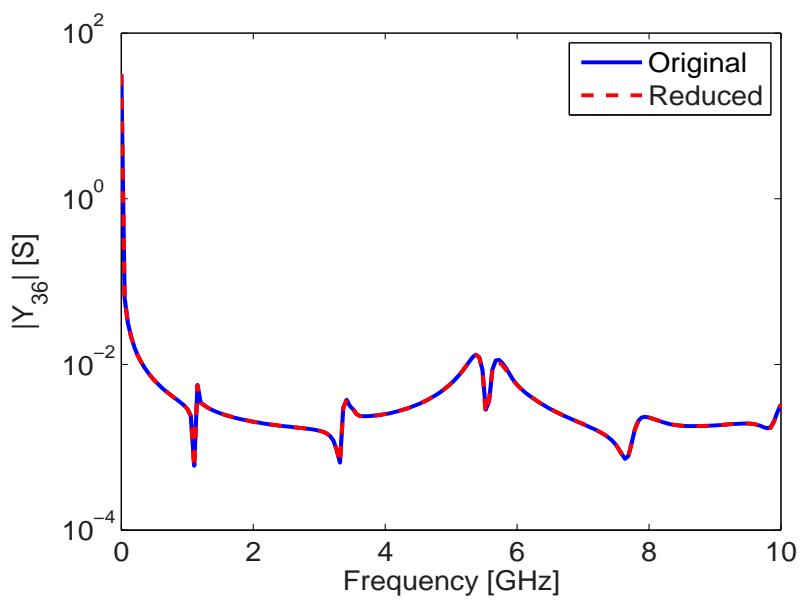

Fig. 6. Magnitude of $\mathbf{Y}_{36}$.

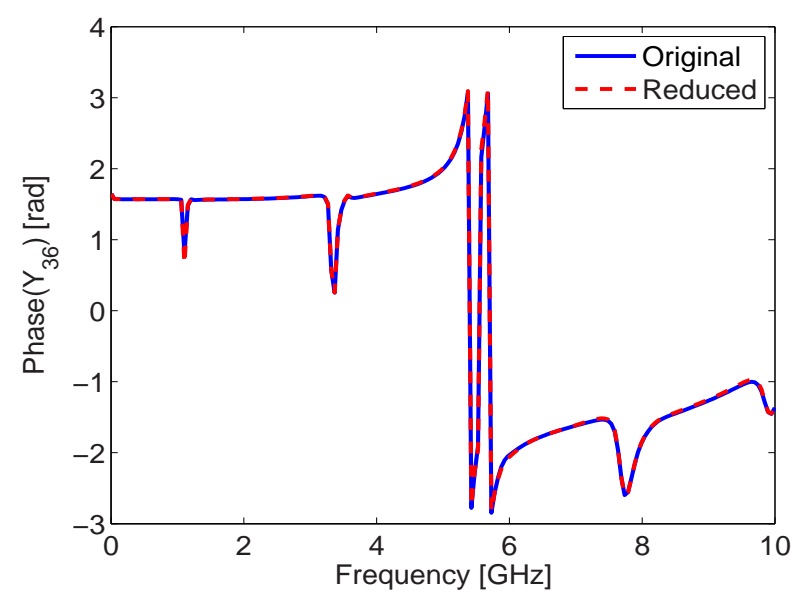

Fig. 7. Phase of $\mathbf{Y}_{36}$. have validated the proposed MOR approach.

\section{ACKNOWLEDGMENT}

This work was supported by the Research Foundation Flanders (FWO).

\section{REFERENCES}

[1] R. F. Harrington, Field Computation by Moment Methods. New York: Macmillan, 1968

[2] A. E. Ruehli, "Equivalent circuit models for three dimensional multiconductor systems," IEEE Trans. Microw. Theory Tech., vol. 22, no. 3, pp. 216-221, Mar. 1974.

[3] A. Odabasioglu, M. Celik, and L. T. Pileggi, "PRIMA: passive reducedorder interconnect macromodeling algorithm," IEEE Trans. Comput.Aided Design Integr. Circuits Syst., vol. 17, no. 8, pp. 645-654, Aug. 1998.

[4] L. Knockaert and D. De Zutter, "Laguerre-SVD reduced-order modeling," IEEE Trans. Microw. Theory Tech., vol. 48, no. 9, pp. 1469-1475, Sep. 2000.

[5] C. A. Balanis, Advanced Engineering Electromagnetics. John Wiley and Sons, New York, 1989

[6] A. E. Ruehli and A. C. Cangellaris, "Progress in the methodologies for the electrical modeling of interconnects and electronic packages," Proc. IEEE, vol. 89, no. 5, pp. 740-771, May 2001.

[7] P. J. Restle, A. Ruehli, S. G. Walker, G. Papadopoulos, "Full-wave PEEC time-domain for the modeling of on-chip interconnects," IEEE Transactions on Computer-Aided Design, vol. 20, no. 7, pp. 877-887, July 2001.

[8] A. E. Ruehli, G. Antonini, J. Esch, J. Ekman, A. Mayo and A. Orlandi, "Non-orthogonal PEEC formulation for time and frequency domain EM and circuit modeling," IEEE Trans. Electromagn. Compat., vol. 45, no. 2, pp. 167-176, May 2003.

[9] J. R. Phillips, E. Chiprout, and D. D. Ling, "Efficient full-wave electromagnetic analysis via model-order reduction of fast integral transforms," in DAC '96: Proceedings of the 33rd annual Design Automation Conference, New York, NY, USA, 1996, pp. 377-382.

[10] J. Cullum, A. Ruehli, T. Zhang, "A method for reduced-order modeling and simulation of large interconnect circuits and its application to PEEC models with retardation," IEEE Trans. Circuits Syst. II, vol. 47, no. 4, pp. 261-373, Apr. 2000.

[11] T. Klemas, L. Daniel, and J. White, "Segregation by primary phase factors: a full-wave algorithm for model order reduction," in Design Automation Conference, 2005. Proceedings. 42nd, Jun. 2005, pp. 943 - 946.

[12] W. Tseng, C. Chen, E. Gad, M. Nakhla, and R. Achar, "Passive order reduction for RLC circuits with delay elements," IEEE Transactions on Advanced Packaging, vol. 30, no. 4, pp. 830 -840, Nov. 2007.

[13] A. E. Ruehli and H. Heeb, "Circuit models for three-dimensional geometries including dielectrics," IEEE Trans. Microw. Theory Tech., vol. 40, no. 7, pp. 1507-1516, Jul. 1992.

[14] C. Ho, A. Ruehli, P. Brennan, "The modified nodal approach to network analysis," IEEE Trans. Circuits Syst., vol. 22, no. 6, pp. 504-509, Jun. 1975.

[15] I. Elfadel and D. Ling, "A block rational Arnoldi algorithm for multipoint passive model-order reduction of multiport RLC networks," in IEEE/ACM International Conference on Computer-Aided Design, Nov. 1997, pp. 66 $-71$

reduced model. Numerical results based on the $\tau$ PEEC method 